\title{
Über organophile Uranglimmer
}

\author{
Von Armin Weiss, Kurt Hartl und Ulrich Hofmann
}

Aus dem Eduard Zintl-Institut für anorganische und physikalische Chemie der Technischen

Hochschule Darmstadt

(Z. Naturforschg. 12 b, 351-355 [1957] ; eingegangen am 18. Februar 1957)

Synthetische und natürliche Uranglimmer der allgemeinen Formel

$$
\mathrm{Me}^{+}\left(\mathrm{H}_{2} \mathrm{O}\right)_{\mathrm{n}}\left\{\mathrm{UO}_{2} \mathrm{XO}_{4}\right\}^{-} \quad(\mathrm{X}=\mathrm{P}, \mathrm{As}, \mathrm{V})
$$

werden beim Ersatz der anorganischen Kationen $\mathrm{Me}^{+}$gegen größere organische Kationen zur eindimensionalen innerkristallinen Quellung in nichtwäßrigen Flüssigkeiten befähigt. Der ausgeprägt organophile Charakter derartiger Uranglimmer eröffnet vielleicht eine neue Möglichkeit zur Aufbereitung von Gesteinen mit geringen Uranglimmer-Gehalten.

Aus der Messung der Schichtabstände ergab sich der Hinweis, daß bei n-Alkylammonium-Ionen bis zu 8 C-Atomen die Alkylketten senkrecht zu den Uranglimmer-Schichten stehen. Von 12 C-Atomen an sind die Alkylketten zunehmend geneigt.

In den gequollenen Uranglimmern liegen geradkettige Quellungsflüssigkeiten mit der Längsachse ihrer Molekel weitgehend parallel zu den n-Alkylammonium-Ionen. Der Schichtabstand ergibt sich aus der Summe der C-Atome von Quellungsflüssigkeit und Alkylammonium-Ion.

In einer früheren Arbeit wurde gezeigt, daß die Uranglimmer zur eindimensionalen innerkristallinen Quellung befähigt sind ${ }^{1}$. Dieses innerkristalline Quellungsvermögen und das Kationen-Austauschvermögen führten dazu, die Formel dieser Verbindungen als

$$
\underbrace{\mathrm{Me}^{+}+x \mathrm{H}_{2} \mathrm{O}}_{\begin{array}{c}
\text { Wasserschicht mit } \\
\text { austauschfähigen } \\
\text { Kationen }
\end{array}} \cdot \underbrace{\left\{\mathrm{UO}_{2} \cdot \mathrm{XO}_{4}\right\}^{-}}_{\begin{array}{c}
\text { Uranglimmer- } \\
\text { Schicht }
\end{array}} \quad(\mathrm{X}=\mathrm{P}, \mathrm{As}, \mathrm{V})
$$

zu formulieren. Als größter Schichtabstand bei der innerkristallinen Quellung wurde nach dem Eintausch von Lysin als Kation in ein allerdings schlecht kristallisiertes Uranylvanadat bei $p_{\mathrm{H}} 4,0$ unter Wasser ein Wert von ca. 17,5 $\AA$ beobachtet.

Neuere Versuche haben nun gezeigt, daß eine sehr viel stärkere Aufweitung des Schichtgitters möglich ist, wenn man als austauschfähige Kationen große organische Ionen, z. B. Alkylammonium-Ionen einlagert.

Die Herstellung solcher Alkylammonium-Uranglimmer kann entweder durch Umsetzung der Oxonium-Uranglimmer ${ }^{2}$ mit den freien Aminen oder durch die direkte Synthese von AlkylammoniumUranglimmern oder durch den Umtausch der austauschfähigen Kationen eines gewöhnlichen Uranglimmers gegen Alkylammonium-Ionen aus den wäßrigen Chloridlösungen derselben erfolgen.

Die Einlagerung der Alkylammonium-Ionen in die Hydronium-Ionen enthaltenden Uranglimmer kann in

1 A. Weiss u. U. Hofmann, Z. Naturforschg. 7 b, 362 [1952]. einfacher Weise durch direkte Einwirkung des freien Amins oder einer alkoholischen oder ätherischen Lösung desselben auf den suspendierten Uranglimmer erfolgen nach

$$
\mathrm{R} \cdot \mathrm{NH}_{2}+\mathrm{H}_{3} \mathrm{O}^{+}\left\{\mathrm{UO}_{2} \mathrm{XO}_{4}\right\}^{-} \rightarrow \mathrm{RNH}_{3}{ }^{+}\left\{\mathrm{UO}_{2} \mathrm{XO}_{4}\right\}^{-}+\mathrm{H}_{2} \mathrm{O} \text {. }
$$

Zur direkten Synthese der Alkylammonium-Uranglimmer wurde in eine halb gesättigte Lösung von Uranylacetat in 40-proz. Äthylalkohol bei $60^{\circ} \mathrm{C}$ eine auf $60^{\circ} \mathrm{C}$ erwärmte Lösung von primärem Alkylammoniumphosphat bzw. -arsenat langsam eingetropft. Das Molverhältnis $\mathrm{UO}_{2}{ }^{2^{+}}: \mathrm{PO}_{4}{ }^{3^{-}}\left(\mathrm{AsO}_{4}{ }^{3-}\right)$ betrug etwa $1,0: 1,1$. Der ausfallende hellgelbe Niederschlag wurde jeweils 2 Stdn. bei $80^{\circ} \mathrm{C}$ am Wasserbad gealtert und mit heißem Wasser und Äthylalkohol gewaschen.

Zum Ionenaustausch wurden jeweils ca. $1 \mathrm{~g}$ des beliebige andere Kationen enthaltenden Uranglimmers in ca. $50 \mathrm{ml}$ einer etwa 5 -proz. wäßrigen oder wäßrigalkoholischen Lösung eines Alkylammonium-Salzes suspendiert und je nach der Teilchengröße und den ursprünglich gebundenen Kationen mehrere Stdn. bis zu mehreren Wochen darunter aufbewahrt. Zur Erzielung eines vollständigen Austausches wurde die Austauschlösung mehrmals erneuert. Da bei höheren Temperaturen die Austausch-Geschwindigkeiten wesentlich größer sind als bei Raumtemperatur, wurden unsere A"rstauschversuche zum größten Teil bei $65^{\circ} \mathrm{C}$ ausgeführt. Führt man diesen Eintausch von AlkylammoniumIonen mit großen Kristallen natürlich vorkommender Uranglimmer durch, dann beobachtet man mit fortschreitendem Alkylammonium-Ionen-Eintausch eine zunehmende makroskopische Aufweitung der Kristalle senkrecht zu den Plättchenebenen.

Die Ergebnisse der röntgenographischen Untersuchung verschiedener n-Alkylammonium-Uranglimmer sind in der Tab. 1 zusammengestellt. 
Zur Messung des Schichtabstandes im getrockneten Zustand waren die Uranglimmer sorgfältig mit Wasser, Alkohol und anschließend mit Äther ausgewaschen und dann in dünnwandigen Glaskapillaren bei ca. $20^{\circ} \mathrm{C}$ längere Zeit evakuiert worden ( $p<1$ Torr). Für die Aufnahmen unter den verschiedenen Flüssig. keiten waren diese in die mit den Uranglimmern beschickten und evakuierten Glaskapillaren aufgesaugt und die abgeschmolzenen Präparate vor der Röntgenaufnahme zur Einstellung des Gleichgewichtes mindestens 24 Stdn. aufbewahrt worden.

Wie aus der Tabelle hervorgeht, nimmt der Schichtabstand mit steigender Kettenlänge der Alkylammonium-Ionen ziemlich gleichmäßig zu. Die Werte für die Arsenate sind meistens etwas größer als die für die Phosphate.

Auf die Anordnung der n-Alkylammonium-Ionen zwischen den Uranglimmer-Schichten gibt die Tab. 2 einen Hinweis. In dieser Tabelle sind in der 1. Spalte die Zusammensetzung der Uranglimmerschicht, in der 2. Spalte das eingelagerte n-AlkylammoniumIon und in der 3. Spalte die analytisch bestimmte Menge desselben in Molen pro Mol der idealisierten Formel $\mathrm{Me}^{+}\left(\mathrm{UO}_{2} \mathrm{XO}_{4}\right)^{-}$aufgeführt. Zur Analyse wurden der C-Gehalt durch Verbrennung und der $\mathrm{N}$-Gehalt nach K jeldahl bestimmt. Spalte 4 enthält den Schichtabstand des sorgfältig ausgewaschenen Alkylammonium-Uranglimmers nach der Trocknung bei $65^{\circ} \mathrm{C}$ und bei Drucken unter 1 Torr, Spalte 5 die durch die Alkylammonium-Ionen bedingte Schichtaufweitung, berechnet aus dem gemessenen Schichtabstand und dem Schichtabstand des wasserfreien Ammonium-Uranglimmers

$$
\begin{aligned}
& d_{\left(\mathrm{NH}_{4} \mathrm{UO}_{2} \mathrm{PO}_{4}\right)}=6,3 \AA \text {; } \\
& d_{\left(\mathrm{NH}_{4} \mathrm{UO}_{2} \mathrm{AsO}_{4}\right)}=6,4 \AA \text {; } \\
& d_{\left(\mathrm{NH}_{4} \mathrm{UO}_{2} \mathrm{VO}_{4}\right)}=6,6 \AA .
\end{aligned}
$$

Die Werte in Spalte 5 geben daher nur die durch die Alkylketten bedingte Schichtaufweitung wieder.

In Spalte 6 sind die Schichtaufweitungen durch die eingelagerten Alkylammonium-Ionen angegeben, wie sie sich theoretisch bei der Annahme einer senkrechten Stellung der gestreckten Alkylketten zu den Schichtebenen nach

$$
d_{\text {theoret. }}=(n-1) \cdot 1,26+3,54 \AA
$$

errechnen (Abb. 1). Dabei bedeutet $n$ die Zahl der C-Atome einer Alkylkette. Ein Vergleich der Werte

\footnotetext{
3 P. Franzen, Clay Minerals Bull. 2, 223 [1955].

4 A. Mehler, Dissertation, Darmstadt 1956.
}

in den Spalten 5 und 6 zeigt, daß die Werte einander recht ähnlich sind und läßt es wahrscheinlich erscheinen, daß die Alkylammonium-Ionen bei geringer Alkylkettenlänge (bis zu $8 \mathrm{C}$-Atomen) mit ihrer Längsachse senkrecht, bei größerer Alkylkettenlänge (12 C-Atome und mehr) zumindest sehr steil zu den Schichtebenen stehen. Die Spalte 7 gibt die Winkel zwischen der Längsachse der Alkylammonium-Ionen und den Schichtebenen an, welche sich aus den Werten der Spalten 5 und 6 errechnen.

Auch die Ergebnisse von Franzen ${ }^{3}$ an Trimethylcetylammonium-Montmorillonit führen $\mathrm{zu}$ einem ähnlichen Modell, wenngleich beim Montmorillonit die Ladungsdichte sehr viel geringer ist, so da 3 im Zwischenschicht-Raum der ungequollenen Proben nur etwa ein Drittel der geometrisch möglichen Positionen durch n-Alkylammonium-Ionen besetzt ist. Die Messungen von Franzen beziehen sich aber auf ein Präparat, welches überschüssiges Alkylammonium-Salz zwischen den Schichten enthielt.

Die Vorstellungen über die Anordnung der Alkylammonium-Ionen in den Uranglimmern stehen auch in Einklang mit den Beobachtungen beim Austausch kleiner anorganischer Kationen gegen große Alkylammonium-Ionen in Montmorillonit ${ }^{4}$, Vermikulit ${ }^{5}$ und den Mineralen der Glimmergruppe ${ }^{6}$. Bei den Silikaten tritt aber außer einer Anordnung mit senkrecht stehenden Alkylketten noch bevorzugt eine solche mit einem Neigungswinkel von $56^{\circ}$ auf.

Für die Anordnung von geradkettigen Quellungsflüssigkeiten zeigt die Abb. 2 eine plausible Annahme, die sich durch die in der Tab. 1 angegebenen Schichtabstände unter den verschiedenen Alkoholen stützen läßt. Der Schichtabstand bei den geradkettigen Quellungsflüssigkeiten wird im wesentlichen durch die Anzahl der C-Atome bestimmt. So beträgt z. B. der Schichtabstand von getrocknetem n-Dodecylammonium-uranylphosphat 22,5 $\AA$, der von n-Hexylammonium-uranylphosphat unter nHexanol 22,6 $\AA$ und unter n-Hexylamin 22,8 $\AA$, der von $\mathrm{n}$-Butylammonium-uranylphosphat unter n-Octanol 22,7 $\AA$ und schließlich der von n-Octylammonium-uranylphosphat unter n-Butylalkohol 22,7 $\AA$. Aus der Tab. 3 lassen sich noch andere derartige Gruppen erkennen.

5 A. Weiss, A. Mehler u. U. Hofmann, Z. Naturforschg. 11 b, 431 [1956].

6 A. Weiss, A. Mehler u. U. Hofmann, Z. Naturforschg. 11 b, 435 [1956]. 


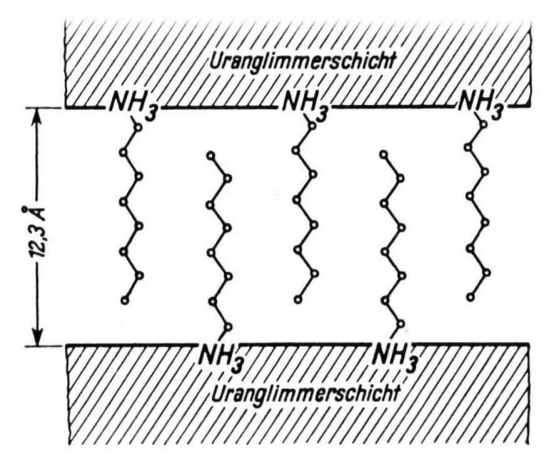

Abb. 1. Idealisierte Darstellung der Anordnung von Alkylammonium-Ionen zwischen den Uranglimmer-Schichten (für Octylammonium-uranylphosphat gezeichnet).

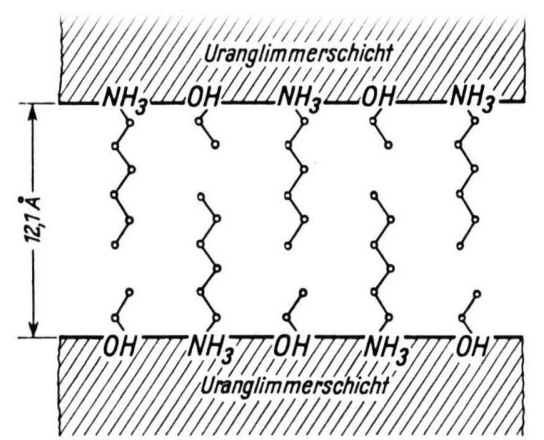

Abb. 2. Idealisierte Darstellung der Anordnung von Alkyl ammonium-Ionen und Quellungsflüssigkeiten zwischen den Uranglimmerschichten (für Hexylammonium-uranylphosphat mit Åthylalkohol als Quellungsflüssigkeit gezeichnet). Der Ubersichtlichkeit halber wurde pro Alkylammoniumion eine Molekel der Quellungsflüssigkeit eingezeichnet.

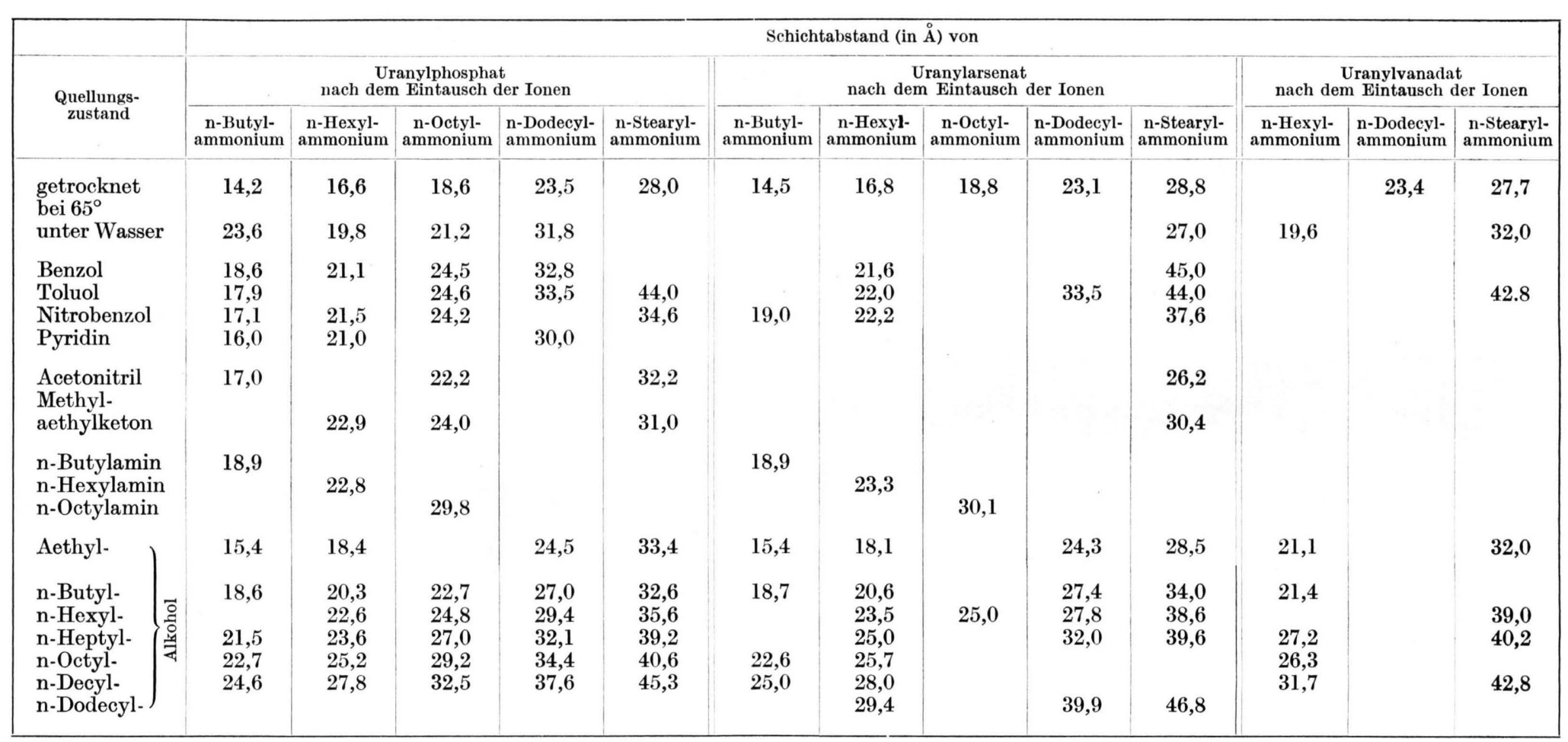

Tab. 1. Schichtabstand von synthetischen Uranglimmern nach der Einlagerung von n-Alkylammonium-Ionen unter verschiedenen Quellungsflüssigkeiten. 


\begin{tabular}{|c|c|c|c|c|c|c|}
\hline 1 & 2 & 3 & 4 & 5 & 6 & 7 \\
\hline $\begin{array}{c}\text { Zusammen- } \\
\text { setzung der } \\
\text { Uran- } \\
\text { glimmer- } \\
\text { Schicht }\end{array}$ & $\begin{array}{c}\text { Eingelagerte } \\
\text { n-Alkyl- } \\
\text { ammonium- } \\
\text { Ionen }\end{array}$ & $\begin{array}{l}\text { Mole Amin } \\
\text { pro Mol der } \\
\text { idealisierten } \\
\text { Formel } \\
\mathrm{UO}_{2} \mathrm{XO}_{4}\end{array}$ & $\begin{array}{c}\text { Schicht- } \\
\text { abstände nach } \\
\text { dem Trocknen } \\
\text { bei } 65^{\circ} \mathrm{C} \\
\left(=d_{\left.65^{\circ} \mathrm{C}\right)}\right. \\
{[\AA]}\end{array}$ & $\begin{array}{c}\text { Schicht- } \\
\text { aufweitg. durch } \\
\text { die Alkvlketten } \\
\begin{array}{c}\left(=d_{65^{\circ}}\right. \\
- \\
\left.-d_{\mathrm{NH}_{4}}\right) * \\
{[\AA]}\end{array}\end{array}$ & $\begin{array}{l}\text { Theor. errechn. } \\
\text { Schicht- } \\
\text { aufweitung } \\
=1.26(n-1) \\
+3.54 \\
{[\AA]}\end{array}$ & $\begin{array}{c}\text { Wahrscheinl. } \\
\text { Winkel zw. der } \\
\text { Längsachse der } \\
\text { Alkylketten u.d. } \\
\text { Schichtebenen } \\
\text { [Grad] }\end{array}$ \\
\hline $\mathrm{UO}_{2} \mathrm{PO}_{4}$ & $\begin{array}{l}\text { Propyl- } \\
\text { Butyl- } \\
\text { Amyl- } \\
\text { Hexyl- } \\
\text { Heptyl- } \\
\text { Octyl- } \\
\text { Dodecyl- } \\
\text { Stearyl- }\end{array}$ & $\begin{array}{l}0,93 \\
1,02\end{array}$ & $\begin{array}{l}13,2 \\
14,2 \\
15,3 \\
16,6 \\
18,2 \\
18,6 \\
23,5 \\
28,0\end{array}$ & $\begin{array}{r}6,9 \\
7,9 \\
9,0 \\
10,3 \\
11,9 \\
12,3 \\
17,2 \\
21,7\end{array}$ & $\begin{array}{r}6,0 \\
7,3 \\
8,5 \\
9,8 \\
11,0 \\
12,3 \\
17,4 \\
24,9\end{array}$ & $\begin{array}{l}90 \\
90 \\
90 \\
90 \\
90 \\
90 \\
82 \\
61\end{array}$ \\
\hline $\mathrm{UO}_{2} \mathrm{AsO}_{4}^{-}$ & $\begin{array}{l}\text { Butyl- } \\
\text { Hexyl- } \\
\text { Octyl- } \\
\text { Dodecyl- } \\
\text { Stearyl- }\end{array}$ & $\begin{array}{l}0,95 \\
\\
0,94 \\
0,93\end{array}$ & $\begin{array}{l}14,5 \\
16,8 \\
18,8 \\
23,1 \\
28,8\end{array}$ & $\begin{array}{r}8,1 \\
10,4 \\
12,4 \\
16,7 \\
22,4\end{array}$ & $\begin{array}{r}7,3 \\
9,8 \\
12,3 \\
17,4 \\
24,9\end{array}$ & $\begin{array}{l}90 \\
90 \\
90 \\
74 \\
66,5\end{array}$ \\
\hline $\mathrm{UO}_{2} \mathrm{VO}_{4}^{-}$ & $\begin{array}{l}\text { Hexyl- } \\
\text { Dodecyl- } \\
\text { Stearyl- }\end{array}$ & & $\begin{array}{l}23,4 \\
27,7\end{array}$ & $\begin{array}{l}16,8 \\
21,1\end{array}$ & $\begin{array}{r}9,8 \\
17,4 \\
24,9\end{array}$ & $\begin{array}{l}75 \\
58\end{array}$ \\
\hline
\end{tabular}

Tab. 2. Neigungswinkel der Alkylketten in synthetischen n-Alkylammonium-Uranglimmern.

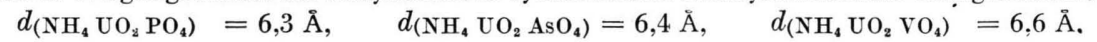

\begin{tabular}{|c|c|c|c|c|c|c|}
\hline \multirow{2}{*}{$\begin{array}{l}\text { Gesamtzahl } \\
\text { der C-A tome } \\
\text { v. Ammonium- } \\
\text { Ion und } \\
\text { Quellungs- } \\
\text { flüssigkeit }\end{array}$} & \multirow{2}{*}{$\begin{array}{l}\text { C-Atomzahl } \\
\text { der einge- } \\
\text { lagerten } \\
\text { n-Alkyl- } \\
\text { ammonium- } \\
\text { Ionen }\end{array}$} & \multirow{2}{*}{$\begin{array}{l}\text { C-Atomzahl der } \\
\text { als Qnellungs- } \\
\text { füssigkeit } \\
\text { eingelagerten } \\
\text { primären } \\
\text { n-Alkohole }\end{array}$} & \multirow{2}{*}{$\begin{array}{l}\text { C-Atomzahl der } \\
\text { als Quellungs- } \\
\text { flüssigkeit } \\
\text { eingelagerten } \\
\text { primären } \\
\text { n-Alkylamine }\end{array}$} & \multicolumn{3}{|c|}{$\begin{array}{c}\text { Schichtabstände (in } \AA \text { ) } \\
\text { von }\end{array}$} \\
\hline & & & & $\begin{array}{l}\text { Uranyl- } \\
\text { phosphat }\end{array}$ & $\begin{array}{l}\text { Uranyl- } \\
\text { arsenat }\end{array}$ & $\begin{array}{l}\text { Uranyl- } \\
\text { vanadat }\end{array}$ \\
\hline 6 & $\begin{array}{l}4 \\
6\end{array}$ & 2 & & $\begin{array}{l}15,4 \\
16,6\end{array}$ & $\begin{array}{l}15,4 \\
16,8\end{array}$ & \\
\hline 8 & $\begin{array}{l}4 \\
4 \\
6 \\
8\end{array}$ & $\begin{array}{l}4 \\
2\end{array}$ & 4 & $\begin{array}{l}18,6 \\
18,9 \\
18,4 \\
18,6\end{array}$ & $\begin{array}{l}18,7 \\
18,9 \\
18,1 \\
18,8\end{array}$ & \\
\hline 12 & $\begin{array}{r}4 \\
6 \\
6 \\
8 \\
12\end{array}$ & $\begin{array}{l}8 \\
6 \\
4\end{array}$ & 6 & $\begin{array}{l}22,7 \\
22,6 \\
22,8 \\
22,7 \\
22,5\end{array}$ & $\begin{array}{l}22,6 \\
23,5 \\
23,3 \\
\\
\\
23,1\end{array}$ & 23,4 \\
\hline 14 & $\begin{array}{r}4 \\
6 \\
8 \\
12\end{array}$ & $\begin{array}{r}10 \\
8 \\
6 \\
2\end{array}$ & & $\begin{array}{l}24,6 \\
25,2 \\
24,8 \\
24,5\end{array}$ & $\begin{array}{l}25,0 \\
25,7 \\
25,0 \\
24,3\end{array}$ & 26,3 \\
\hline 16 & $\begin{array}{r}4 \\
6 \\
8 \\
8 \\
12\end{array}$ & $\begin{array}{r}12 \\
10 \\
8 \\
\\
4\end{array}$ & 8 & $\begin{array}{l}27,8 \\
29,2 \\
29,8 \\
27,0\end{array}$ & $\begin{array}{l}28,0 \\
30,1 \\
27,4\end{array}$ & 31,7 \\
\hline 18 & $\begin{array}{r}6 \\
8 \\
12 \\
18\end{array}$ & $\begin{array}{r}12 \\
10 \\
6\end{array}$ & & $\begin{array}{l}32,5 \\
29,4 \\
28,0\end{array}$ & $\begin{array}{l}29,4 \\
\\
27,8 \\
28,8\end{array}$ & 27,7 \\
\hline
\end{tabular}

Tab. 3. Einfluß der C-Atomzahl bei geradkettigen Alkylammonium-Ionen und geradkettigen Quellungsflüssigkeiten auf den Schichtabstand der verschiedenen synthetischen Uranglimmer. 


\begin{tabular}{|c|c|c|}
\hline Mineral & idealisierte Formel & $\begin{array}{c}\text { Schichtabstand } \\
\text { unter der } \\
\text { Eintauschlösung } \\
{[\AA]}\end{array}$ \\
\hline $\begin{array}{l}\text { Autunit } \\
\text { (Cornwall) }\end{array}$ & \multirow{3}{*}{$\mathrm{Ca}\left(\mathrm{UO}_{2} \mathrm{PO}_{4}\right)_{2} \cdot n \mathrm{H}_{2} \mathrm{O}$} & 33,8 \\
\hline $\begin{array}{l}\text { Autunit } \\
\text { (Autun) }\end{array}$ & & 33,8 \\
\hline $\begin{array}{l}\text { Kalkuranglimmer } \\
\text { (Grube Weißer Hirsch, } \\
\text { Schneeberg/Sachsen) }\end{array}$ & & 32,4 \\
\hline $\begin{array}{l}\text { Torbernit } \\
\text { (Cornwall) }\end{array}$ & \multirow{2}{*}{$\mathrm{Cu}\left(\mathrm{UO}_{2} \mathrm{PO}_{4}\right)_{2} \cdot n \mathrm{H}_{2} \mathrm{O}$} & 32,4 \\
\hline $\begin{array}{l}\text { Torbernit } \\
\text { (Fichtelgeb.) }\end{array}$ & & 32,4 \\
\hline $\begin{array}{l}\text { Zeunerit } \\
\text { (Grube Weißer Hirsch, } \\
\text { Schneeberg/Sachsen) }\end{array}$ & $\mathrm{Cu}\left(\mathrm{UO}_{2} \mathrm{AsO}_{4}\right)_{2} \cdot n \mathrm{H}_{2} \mathrm{O}$ & 32,5 \\
\hline $\begin{array}{l}\text { Trögerit } \\
\text { (Schneeberg/Sachsen) }\end{array}$ & $\mathrm{UO}_{2}\left(\mathrm{UO}_{2} \mathrm{AsO}_{4}\right)_{2} \cdot n \mathrm{H}_{2} \mathrm{O}$ & 33,0 \\
\hline $\begin{array}{l}\text { Carnotit } \\
\text { (Utah) }\end{array}$ & $\mathrm{KUO}_{2} \mathrm{VO}_{4}$ & 46,8 \\
\hline
\end{tabular}

Tab. 4. Schichtabstände natürlicher Uranglimmer nach dem Eintausch von n-Stearylammonium-Ionen unter der Eintauschlösung (wäßrige Stearylammoniumchlorid-Lösung).

Mit steigender Kettenlänge des eingelagerten normalen primären Alkohols steigt der Schichtabstand nicht gleichmäßig an, auch wenn man nur Alkohole mit gerader C-Atomzahl betrachtet. Es erscheint plausibel, $\mathrm{da} ß$ solche kleinen Unregelmäßigkeiten durch die aus den Uranglimmer-Schichten herausragenden Sauerstoffatome der gestreckten Uranylionen verursacht werden.

Bei der Einlagerung verschiedener aromatischer Verbindungen als Quellungsflüssigkeit wurde, wenn man von der Einlagerung in StearylammoniumUranglimmer absieht, die Schichtaufweitung als unabhängig von den Substituenten am Benzolkern gefunden.

Aromatische Amine, als Ammoniumionen in den Zwischenschicht-Raum der verschiedenen Uranglimmer eingelagert, können bei Zutritt von Luftsauerstoff zwischen den Schichten oxydativ verändert werden. Entsprechend dem schwach basischen Charakter der aromatischen Amine können diese Proben in wäßriger Suspension hydrolysieren.

Die Einlagerung großer organischer Kationen gelingt auch bei den in der Natur vorkommenden Uranglimmern. Bei solchen natürlichen Uranglimmern sind die Kationen im Zwischenschicht-Raum z. T. bereits bei den grubenfeuchten Proben nicht mehr gegen andere anorganische Kationen austauschbar und die Schichten bleiben unabhängig vom Wassergehalt der Umgebung in einer Lage fixiert, z. T. geht die Fähigkeit zum Kationenaustausch mit kleinen Kationen und zur innerkristallinen Quellung bereits beim gelinden Erwärmen praktisch irreversibel verloren. Sogar bei derartigen Proben gelingt der Eintausch von AlkylammoniumIonen. Auffallend ist allerdings, daß der Carnotit unter der Eintauschlösung einen sehr viel größeren Schichtabstand zeigt als die anderen untersuchten Uranglimmer.

Während die natürlichen Uranglimmer von nichtwäßrigen Flüssigkeiten nur schlecht benetzt werden, werden die Alkylammonium-Ionen oder z. B. auch Alkylpyridinium-Ionen enthaltenden Uranglimmer von den meisten nichtwäßrigen Flüssigkeiten sehr gut benetzt. Fügt man zum Beispiel zu einer wäßrigen Suspension eines große organische Kationen enthaltenden Uranglimmers nur einige Tropfen Benzol oder Toluol, so geht der Uranglimmer praktisch quantitativ in die Benzolschicht. Die starke Volumzunahme beim Eintausch der großen organischen Kationen führt auch häufig dazu, daß dabei an der Oberfläche fest anhaftende Verunreinigungen an Muttergestein von den Uranglimmer-Plättchen abspringen. Diese Eigenschaft und der organophile Charakter bieten vielleicht eine neue Möglichkeit zur Flotation und damit zur Aufbereitung von Gesteinen mit nur geringen Uranglimmer-Gehalten. 